PESQUIMAT, Revista de la F.C.M. de la

Universidad Nacional Mayor de San Marcos

Vol. XV Nº1, pp. 68-78, Lima - Perú, Marzo 2013

\title{
MÉTODO DEL PUNTO PROXIMAL Y SUS APLICACIÓN A MODELOS ECONÓMICOS
}

\author{
Lucy De la Cruz ${ }^{4}$ y Erik Papa Quiroz ${ }^{5}$
}

Resumen: El objetivo de este trabajo es estudiar la convergencia de una extensión del método del punto proximal para minimizar una clase de funciones no convexas sobre el ortante no negativo y dar algunas aplicaciones del método en la solución de modelos económicos que aparecen en microeconomía.

Los procedimientos utilizados fueron la recopilación de información en revistas científicas y textos especializados, el estudio de los mismos y finalmente el uso de herramientas matemáticas para estudiar la convergencia de la sucesión del método. Los resultados del estudio nos muestran que, bajo algunas hipótesis adecuadas, las iteraciones generadas por el método están bien definidas y la sucesión converge débilmente a un punto de KKT.

Palabras claves: Métodos proximales, ortante no negativo, distancia generalizada, funciones no convexas.

Abstract: The aim of this work is to study the convergence of a extension of the proximal point method for minimizing a class of nonconvex functions on the nonnegative orthant and give some applications of the method in the solution of economics models which appears in microeconomy.

The used procedures were the collection of information in scientific journals and specialized books, the study of the same and finally the use of mathematical tools to study the convergence of the sequence of the method. The results show that, under some appropriate assumptions, the iterations generated by the method are well defined and the sequence converges weakly to a KKT point.

Keywords: Proximal point methods, separable convex problems, nonnegative orthant, proximal distances, convex functions.

Introducción En este trabajo de investigación se analiza la convergencia de una extensión del método del punto proximal para solucionar problemas de minimización cuando la función objetivo es no convexa.

Consideremos el problema (PR):

$$
\min \{f(x): x \geq 0\},
$$

donde $f: \mathbb{R}^{n} \rightarrow \mathbb{R} \cup\{+\infty\}$ es una función propia, acotada inferiormente y semicontinua inferior y $x \geq 0$ significa que $x_{i} \geq 0, \forall i=1, \ldots, n$. La clase de métodos de punto proximal interior que resuelve (PR) genera una sucesión $\left\{x^{k}\right\}$ dada por $x^{0} \in \mathbb{R}_{++}^{n}$ (punto arbitrario) y

$$
x^{k} \in \arg \min _{x \geq 0}\left\{f(x)+\lambda_{k} d\left(x, x^{k-1}\right)\right\},
$$

donde $\lambda_{k}$ es un parámetro positivo y $d$ es una función que satisface algunas propiedades deseables que se asemejan a una distancia euclidiana pero que exigen que las iteraciones se mantengan en el interior del conjunto de restricciones. Algunos ejemplos de $d$ basados en este trabajo son:

\footnotetext{
${ }^{4}$ Universidad Nacional Mayor de San Marcos, dlclucy@hotmail.com

${ }^{5}$ Universidad Nacional Mayor de San Marcos, erikpapa@gmail.com
} 
- Distancias de Bregman:

$$
d(x, y)=D_{h}(x, y):=h(x)-h(y)-\langle\nabla h(y), x-y\rangle,
$$

donde $h: \mathbb{R}^{n} \rightarrow \mathbb{R}$ es una función de Bregman.

- Distancias $\varphi$-divergencias:

$$
d_{\varphi}(x, y)=\sum_{i=1}^{n} y_{i} \varphi\left(\frac{x_{i}}{y_{i}}\right)
$$

donde $\varphi: \mathbb{R}_{+} \rightarrow(-\infty,+\infty)$ es una función que satisface algunas propiedades de convexidad y diferenciabilidad.

- Distancias homogéneas de segundo orden:

$$
d_{\varphi}(x, y)=\sum_{i=1}^{n} y_{i}^{2} \phi\left(\frac{x_{i}}{y_{i}}\right),
$$

donde $\phi(t)=\mu \varphi(t)+\frac{\nu}{2}(t-1)^{2}$ con $\mu, \nu>0$ y $\varphi$ es una función definida en el ítem anterior. Asumiendo que $f$ es una función propia, semicontinua inferior, convexa, dom $f \cap \mathbb{R}_{++}^{n} \neq \emptyset$ y $\left\{\lambda_{k}\right\}$ satisface

$$
\sum_{k=1}^{+\infty} \frac{1}{\lambda_{k}}=+\infty
$$

y usando la distancia proximal, Auslender y Teboulle [1] probaron que $\lim _{k \rightarrow+\infty} f\left(x^{k}\right)=\operatorname{ínf}\{f(x)$ : $x \geq 0\}$. Además, si el conjunto de soluciones óptimas es no vacio, entonces $\left\{x^{k}\right\}$ converge a una solución óptima del problema (PR).

Existe un gran dominio de aplicaciones de optimización cuasi-convexa en el área de las ciencias y la ingeniería tales como: la teoría económica, la teoría de la decisión, la teoría de control, que motivan a estudiar este tema. En particular, este trabajo está motivado a la aplicación del método a modelos de microeconomía.

En el marco teórico presentamos, algunas notaciones, recordamos algunas definiciones y resultados de subgradientes regulares y subgradientes generales.

En el desarrollo del trabajo de investigación, presentamos una extensión del algoritmo proximal en el ortante no negativo para minimizar funciones no convexas y estudiaremos su convergencia. Finalmente mostramos algunos modelos donde el algoritmo estudiado puede ser empleado.

El artículo está organizado de la siguiente manera: En la sección 2 presentamos la metodología empleada en el estudio de investigación. En la sección 3 mostramos los resultados del trabajo, los cuales están divididas en subsecciones que detallamos a seguir: en la subsección 3.1 recordamos algunos elementos básicos de análisis convexo, en la subsección 3.3 introducimos la clefinición de distancias generalizadas, en la subsección 3.4 presentamos el método propuesto. En la sección 5 presentamos las discusiones del trabajo. En la sección 6 los agradecimientos respectivos y finalmente presentamos las referencias bibliográficas empleadas para esta investigación.

\section{Metodología}

La metodología de trabajo fue dividida en las siguientes partes:

\subsection{El universo}

El universo de nuestra investigación es la optimización no convexa en el espacio euclidiano. La optimización no convexa es una línea de la matemática aplicada que estudia el problema de hallar los valores mínimos o máximos de funciones no convexas sujetas a restricciones que forman un conjunto convexo. 
2.2 Técnicas de recopilación de datos Para el inicio de la investigación fue necesario una búsqueda de información científica en revistas publicadas, uso de bibliotecas y hemerotecas especializadas.

2.3 Técnicas descriptivas para la contrastación o demostración de las hipótesis La metodología usada en esta etapa fue un enfoque de tipo inductivo-deductivo de los resultados de recientes investigaciones, con la finalidad de adaptarlo a nuestros requerimientos y que nos llevó a la obtención de los resultados planteados en los objetivos de la investigación.

\section{Resultados}

\section{Algunos Resultados del Análisis Convexo}

A lo largo de este trabajo $\mathbb{R}^{n}$ es el espacio euclideano dotado con el producto interno canónico $\langle$,$\rangle y la norma en x$ dada por $\|x\|:=\langle x, x\rangle^{1 / 2}$.

Definición 3.1 Dada una función $f: \mathbb{R}^{n} \rightarrow \mathbb{R} \cup\{ \pm \infty\}$

1. El dominio de $f$ es definido por domf $:=\left\{x \in \mathbb{R}^{n}: f(x)<+\infty\right\}$;

2. f es llamada una función propia si :

a) $\operatorname{dom} f \neq \emptyset$;

b) $\forall x \in \operatorname{dom} f: f(x)>-\infty$

3. $f$ es semicontinua inferior en $\bar{x}$ si $: \forall\left\{x^{l}\right\} \subset \mathbb{R}^{n}$ tal que $\lim _{l \rightarrow+\infty} x^{l}=\bar{x}$ se tiene que

$$
f(\bar{x}) \leq \liminf _{l \rightarrow+\infty} f\left(x^{l}\right)
$$

Decimos que $f$ es una función semicontínua inferior si es semicontínua inferior en cada punto de $\mathbb{R}^{n}$.

Definición 3.2 Sea $f: \mathbb{R}^{n} \rightarrow \mathbb{R} \cup\{ \pm \infty\}$ una función propia, convexa. Decimos que y $\in \mathbb{R}^{n}$ es un subgradiente de $f$ en un punto $x \in \mathbb{R}^{n}$ si

$$
f(z) \geq f(x)+\langle y, z-x\rangle, \quad \forall z \in \mathbb{R}^{n} .
$$

El conjunto de todos los subgradientes de $f$ en $x$ se llama el subdiferencial de $f$ en $x$; lo denotaremos por $\partial^{F} f(x)$.

Teorema 3.1 (El subdiferencial de una función convexa) Sea $f: \mathbb{R}^{n} \rightarrow \mathbb{R} \cup\{ \pm \infty\}$ una función propia, semicontinua inferior y convexa. Entonces para todo $x \in \mathbb{R}^{n}$, el conjunto $\partial^{F} f(x)$ es convexo, compacto y no vacio. Además, para todo $d \in \mathbb{R}^{n}$, se tiene

$$
f^{\prime}(x ; d)=\operatorname{máx}_{y \in \partial^{F f}(x)}\langle y, d\rangle .
$$

Demostración. Ver Makela [3], pg. 13.

Teorema 3.2(Condición de optimalidad para minimización) Sea $f: \mathbb{R}^{n} \rightarrow \mathbb{R}$ una función convexa y $D \subset \mathbb{R}^{n}$ un conjunto convexo. Entonces $\bar{x} \in \mathbb{R}^{n}$ es un minimizador de $f$ en D si, y sólo si,

$$
\exists y \in \partial^{F} f(\bar{x}) \quad \text { tal que } \quad\langle y, x-\bar{x}\rangle \geq 0 \quad \forall x \in D .
$$


En particular, $\bar{x}$ es un minimizador de $f$ en $\mathbb{R}^{n}$ si, y sólo si,

$$
0 \in \partial^{F} f(\bar{x})
$$

Demostración. Ver Izmailov y Solodov [2], pg. 169.

\subsection{Subdiferencial de Funciones no convexas}

Proposición 3.1 Sea $f: \mathbb{R}^{n} \rightarrow \mathbb{R} \cup\{ \pm \infty\}$ una función propia. $f$ es semicontínua inferior si, y sólo si, el conjunto de nivel $L_{f}(\alpha)=\left\{x \in \mathbb{R}^{n}: f(x) \leq \alpha\right\}$ es un conjunto cerrallo para todo $\alpha \in \mathbb{R}$.

Demostración. Ver Izmailov y Solodov [2], pg. 14.

Proposición 3.2 Sea $X \subset \mathbb{R}^{n}$ un conjunto no vacio y $f: X \rightarrow \mathbb{R}$ una función semicontínua inferior y coerciva en $X$, entonces existe un punto de minimo global de $f$ en $X$.

Demostración. Ver Izmailov and Solodov [2], pg. 14.

Definición 3.3 Sea $f: \mathbb{R}^{n} \rightarrow \mathbb{R} \cup\{+\infty\}$ una función propia. Para cada $x \in \operatorname{dom} f$, el conjunto de subgradientes regulares (también llamado Subdiferencial de Fréchet) de $f$ en $x$, denotado por $\widehat{\partial} f(x)$, es el conjunto de vectores $s \in \mathbb{R}^{n}$ tal que

$$
\liminf _{y \neq x, y \rightarrow x} \frac{1}{\|x-y\|}[f(y)-f(x)-\langle s, y-x\rangle] \geq 0 .
$$

Si $x \notin$ domf entonces $\widehat{\partial} f(x)=\emptyset$.

Observemos que esta definición es equivalente a la siguiente

$$
f(y) \geq f(x)+\langle s, y-x\rangle+o(\|x-y\|),
$$

donde

$$
\lim _{y \rightarrow x} \frac{o(\|x-y\|)}{\|x-y\|}=0 .
$$

Proposición 3.3 Para cada $x \in \mathbb{R}^{n}, \widehat{\partial} f(x)$ es un conjunto cerrado y convexo.

Demostración. Ver Rockafellar y Wets [4], Teorema 8.6, pg. 302.

Definición 3.4 Sea $f: \mathbb{R}^{n} \rightarrow \mathbb{R} \cup\{+\infty\}$ una función propia. El conjunto de súbgradientes generales (también llamados subdiferencial en el limite) de $f$ en $x \in \mathbb{R}^{n}$, denotado por $\partial f(x)$, es definido de la siguiente manera

$$
\partial f(x):=\left\{s \in \mathbb{R}^{n}: \exists x^{l} \rightarrow x, f\left(x^{n}\right) \rightarrow f(x), s^{l} \in \widehat{\partial} f\left(x^{l}\right) \rightarrow s\right\} .
$$

Proposición 3.4 Las siguientes propiedades son verdaderas.

a. $\widehat{\partial} f(x) \subset \partial f(x)$,para todo $x \in \mathbb{R}^{n}$;

b. Si $f$ es diferenciable en $\bar{x}$ entonces $\widehat{\partial} f(\bar{x})=\{\nabla f(\bar{x})\}$, además $\nabla f(\bar{x}) \in \partial f(\bar{x})$; 
c. Si $f$ es continuamente diferenciable en una vecindad de $x$, entonces

$$
\widehat{\partial} f(x)=\partial f(x)=\{\nabla f(x)\},
$$

d. Si $g=f+h$ con $f$ finito en $\bar{x} y h$ es continuamente diferenciable en una vecindad de $\bar{x}$ entonces

$$
\begin{aligned}
& \widehat{\partial} g(\bar{x})=\widehat{\partial} f(\bar{x})+\nabla h(\bar{x}), \\
& \partial g(\bar{x})=\partial f(\bar{x})+\nabla h(\bar{x}) .
\end{aligned}
$$

Demostración. Ver Rockafellar y Wets [4], pg 304.

Proposición 3.5 Para cualquier función propia y convexa $f: \mathbb{R}^{n} \rightarrow \mathbb{R} \cup\{ \pm \infty\}$ y cualquier punto $\bar{x} \in \operatorname{domf}$ se tiene

$$
\partial^{F} f(\bar{x})=\{v: f(x) \geq f(\bar{x})+\langle v, x-\bar{x}\rangle, \text { para todo } \mathrm{x}\}=\widehat{\partial} f(\bar{x})
$$

Demostración. Ver Rockafellar y Wets [5], pg 308

Para trabajar con problemas de minimización necesitamos la siguiente definición generalizada.

Definición 3.5 Sea $f: \mathbb{R}^{n} \rightarrow \mathbb{R} \cup\{+\infty\}$ una función propia. Un punto $x \in$ domf es llamado punto crítico generalizado de $f$ si $0 \in \partial f(x)$.

Teorema 3.3 Si una función propia $g: \mathbb{R}^{n} \rightarrow \mathbb{R} \cup\{+\infty\}$ tiene un minimo local en $\bar{x}$ entonces $0 \in \widehat{\partial} g(\bar{x})$ y por lo tanto,

$$
0 \in \partial g(\bar{x})
$$

Demostración. Como $g$ tiene un mínimo local en $\bar{x}$ entonces existe $\delta>0$ tal que

$$
g(\bar{x}) \leq g(y) \quad \forall y \in V_{\delta}(\bar{x})
$$

por otro lado definiendo $o(\|y-\bar{x}\|)=\min \{0, g(y)-g(\bar{x})\}$ obtenemos que

$$
g(y)-g(\bar{x}) \geq 0+o(\|y-\bar{x}\|)
$$

luego, $g(y) \geq g(\bar{x})+\langle 0, y-\bar{x}\rangle+o(\|y-\bar{x}\|)$, además dado $\epsilon>0$ por $(1,1)$ existe $\delta>0$ tal que $0<\|y-\bar{x}\|<\delta$ implica que $\frac{o(\|y-\bar{x}\|)}{\|y-\bar{x}\|}=0<\epsilon$ asi,

$$
\lim _{y \rightarrow \bar{x}} \frac{o(\|\bar{x}-y\|)}{\|\bar{x}-y\|}=0
$$

entonces $0 \in \widehat{\partial} g(\bar{x})$, y por la proposición 1.2.5, a. obtenemos que $0 \in \partial g(\bar{x})$.

\subsection{Dístancia Generalizada}

En esta subsección presentamos una variante de la definición de distancia proximal y distancia proximal inducida, introducida por Auslender y Teboulle [1], pero adaptado para el conjunto $\mathbb{R}_{+}^{n}$ que es la restricción del problema estudiado. Definición 3.6 Una función $d: \mathbb{R}^{n} \times \mathbb{R}^{n} \rightarrow \mathbb{R}_{+} \cup\{+\infty\}$ es denominada distancia proximal en $\mathbb{R}_{++}^{n}$ si para cada $y \in \mathbb{R}_{++}^{n}$ se satisface las siguientes propiedades: 
i. $d(\cdot, y)$ es propia, semicontinua inferior, estrictamente convexa y continuamente diferenciable en $\mathbb{R}_{++}^{n}$;

ii. dom $d(\cdot, y) \subset \mathbb{R}_{+}^{n}$ y dom $\partial_{1} d(\cdot, y)=\mathbb{R}_{++}^{n}$, donde $\partial_{1} d(\cdot, y)$ denota el clásico subdiferencial de la función $d(\cdot, y)$ con respecto a la primera variable;

iii. $d(\cdot, y)$ es coerciva en $\mathbb{R}^{n}$ (es decir, $\lim _{\|u\| \rightarrow \infty} d(u, y)=+\infty$ ).

iv. $d(y, y)=0$.

Denotamos por $D\left(\mathbb{R}_{++}^{n}\right)$ a la familia de funciones satisfaciendo esta definición.

La propiedad i. es necesaria para preservar la convexidad de $d(\cdot, y)$, la propiedad ii forzará que la iteración del método proximal permanezca en $\mathbb{R}_{++}^{n}$, y la propiedad iii será usada para garantizar las existencias de las iteraciones proximales del método propuesto. Para cada $y \in \mathbb{R}_{++}^{n}, \nabla_{1} d(\cdot, y)$ denota el gradiente de la función $d(\cdot, y)$ con respecto a la primera variable. Notemos que por definición $d(\cdot, \cdot) \geq 0$ y de iv. el mínimo global de $d(\cdot, y)$ se obtiene en $y$, y así tenemos que $\nabla_{1} d(y, y)=0$.

Definición 3.7 Dado $d \in D\left(\mathbb{R}_{++}^{n}\right)$, una función $H: \mathbb{R}^{n} \times \mathbb{R}^{n} \rightarrow \mathbb{R}_{+} \cup\{+\infty\}$ es denominada distancia proximal inducida por d si $H$ tiene valores finitos en $\mathbb{R}_{++}^{n} \times \mathbb{R}_{++}^{n}$ y para cada $a, b \in \mathbb{R}_{++}^{n}$ se satisface

(Ii) $H(a, a)=0$.

(Iii) $\left\langle c-b, \nabla_{1} d(b, a)\right\rangle \leq H(c, a)-H(c, b)-H(b, a), \quad \forall c \in \mathbb{R}_{++}^{n}$.

Escribiremos $(d, H) \in \mathscr{F}\left(\mathbb{R}_{++}^{n}\right)$ a la distancia proximal y distancia proximal inducida que satisface las premisas de la Definición 3.7.

Denotemos por $(d, H) \in \mathscr{F}\left(\mathbb{R}_{+}^{n}\right)$ si existe una función $H$ tal que:

(Iiii) $H$ tiene valores finitos en $\mathbb{R}_{+}^{n} \times \mathbb{R}_{++}^{n}$ satisfaciendo (Ii) y (Iii), para cada $c \in \mathbb{R}_{+}^{n}$.

(Iiv) Para cada $c \in \mathbb{R}_{+}^{n}, H(c, \cdot)$ tiene conjuntos de nivel acotados en $\mathbb{R}_{++}^{n}$.

Finalmente, escribiremos $(d, H) \in \mathscr{F}_{+}\left(\mathbb{R}_{+}^{n}\right)$ si

(Iv) $(d, H) \in \mathscr{F}\left(\mathbb{R}_{+}^{n}\right)$.

(Ivi) $\forall y \in \mathbb{R}_{+}^{n}$ y $\forall\left\{y^{k}\right\} \subset \mathbb{R}_{++}^{n}$ acotada con $\lim _{k \rightarrow+\infty} H\left(y, y^{k}\right)=0$, entonces $\operatorname{lím}_{k \rightarrow+\infty} y^{k}=y$.

(Ivii) $\forall y \in \mathbb{R}_{+}^{n}, \mathrm{y} \forall\left\{y^{k}\right\} \subset \mathbb{R}_{++}^{n}$ tal que $\operatorname{lím}_{k \rightarrow+\infty} y^{k}=y$, entonces $\lim _{k \rightarrow+\infty} H\left(y, y^{k}\right)=0$.

El resultado principal del método propuesto será cuando $(d, H) \in \mathscr{F}_{+}\left(\mathbb{R}_{+}^{n}\right)$. Varios ejemplos de distancias proximales que se ajusten a las definiciones anteriores, por ejemplo distancias Bregman, distancias proximal sobre la base de $\phi$ - divergencias, distancias auto-proximales y las distancias sobre la base de distancias proximales homogéneas de segundo orden, fueron dadas por Auslender y Teboulle [1].

\subsection{Método Proximal}

Estamos interesados en resolver el problema

$$
\operatorname{mín}\{f(x): x \geq 0\}
$$

donde $f: \mathbb{R}^{n} \rightarrow \mathbb{R} \cup\{+\infty\}$ es una función propia tal que $\operatorname{dom} f \cap \mathbb{R}_{+}^{n} \neq \emptyset$ y $x \geq 0$ significa que cada componente de $x, x_{i}$, es no negativo.

En esta subsección proponemos una extensión del método de punto proximal con una distancia proximal para resolver el problema planteado. 


\section{Algoritmo}

Dados una sucesión de parámetros positivos $\left\{\lambda_{k}\right\}$ y un punto inicial

$$
x^{0} \in \mathbb{R}_{++}^{n} .
$$

Para cada $k=1,2, \ldots$, si $0 \in \widehat{\partial} f\left(x^{k-1}\right)$, entonces parar. Caso contrario, encontrar $x^{k} \in \mathbb{R}^{n}$ tal que

$$
0 \in \widehat{\partial}\left(f(\cdot)+\lambda_{k} d\left(\cdot, x^{k-1}\right)\right)\left(x^{k}\right),
$$

donde $d$ es una distancia proximal tal que $(d, H) \in F_{+}\left(\mathbb{R}_{+}^{n}\right)$.

Observación 3.1 Observe que el algoritmo propuesto es una extensión (para funciones no convexas) del método de punto proximal. En realidad, si $f$ es convexa entonces la iteraciones se convierten en

$$
x^{k}=\arg \min _{x \geq 0}\left\{f(x)+\lambda_{k} d\left(x, x^{k-1}\right)\right\}
$$

Observación 3.2 Como se está interesado en resolver (4,1) cuando $f$ es no convexa es

importante observar que en el algoritmo sólo necesitamos, en cada iteración, encontrar un punto estacionario (y no un minimo global) de la función regularizadora $f(\cdot)+\lambda_{k} d\left(\cdot, x^{k-1}\right.$ ) asi creemos que los algoritmos locales se pueden utilizar satisfactoriamente en cada iteración. Observación 3.3 De (3.7), y como $\left(\lambda_{k} / 2\right) d\left(\cdot, x^{k-1}\right)$, es continuamente diferenciable, aplicando

la Proposición 1.2.5,d obtenemos que

$$
0 \in \widehat{\partial} f\left(x^{k}\right)+\lambda_{k} \nabla_{1} d\left(x^{k}, x^{k-1}\right) .
$$

Así, existe $g^{k} \in \widehat{\partial} f\left(x^{k}\right)$ tal que

$$
g^{k}=-\lambda_{k} \nabla_{1} d\left(x^{k}, x^{k-1}\right)
$$

\subsection{Resultados de Convergencia}

Teorema 3.4 Sea $f$ es una función acotada inferiormente y semicontinua inferior, entonces la sucesión $\left\{x^{k}\right\}$, generada por el método proximal está bien definida y además $x^{k} \in \mathbb{R}_{++}^{n}$.

Demostración. Como $f$ es semicontinua inferior y acotada inferiormente y cle $d(\cdot, y)$ es coerciva aplicando la Proposición 1.2.2 $f(x)+\lambda_{k} d\left(x, x^{k-1}\right)$ es semicontinua inferior y coerciva luego se garantiza la existencia de $\left\{x^{k}\right\}$. La positividad de $x^{k}$ es inmediata de la clefinición de $d$.

Hipótesis A. $f: \mathbb{R}^{n} \rightarrow \mathbb{R} \cup\{+\infty\}$ es una función propia semicontinua inferior y acotada inferiormente.

Para obtener más información en el punto limite restringimos la clase de: clistancias proximales a una clase especial de distancias proximales separables, esto es,

$$
d(x, y)=\sum_{i=1}^{n} d_{i}\left(x_{i}, y_{i}\right),
$$


donde $d_{i}: \mathbb{R} \times \mathbb{R} \rightarrow \mathbb{R} \cup\{+\infty\}$.

Definamos también:

$$
J(\bar{x})=\left\{i \in\{1,2, \ldots, n\}: \bar{x}_{i}>0\right\} .
$$

Teorema 3.5 Supongamos que la hipótesis $A$ es satisfecha, $d$ es separable. Si $0<\lambda_{k}<\bar{\lambda}$, para todo $k$ y algún $\bar{\lambda}>0$, entonces la sucesión $\left\{x^{k}\right\}$ converge débilmente a un punto $\bar{x}$ que satisface:

i). $\bar{x}_{i} \geq 0, \quad \forall i=1,2, \ldots, n$;

ii). $\liminf _{k \rightarrow+\infty} g_{i}^{k} \geq 0, \quad \forall i \in J(\bar{x})$

iii). $\liminf _{k \rightarrow+\infty} x_{i}^{k} g_{i}^{k} \geq 0, \quad \forall i \in J(\bar{x})$.

Demostración. Sea $\bar{x} \in \mathbb{R}_{+}^{n}$ tal que

$$
\lim _{k \rightarrow+\infty} x^{k}=\bar{x}
$$

Como $x^{k} \geq 0$, la condición i) se satisface facilmente. Probaremos ii).

De la Observación 3.3.1 tenemos

$$
\lim _{k \rightarrow+\infty} g_{i}^{k}=\lim _{k \rightarrow+\infty}-\lambda_{k} \frac{\partial d_{i}}{\partial x_{i}}\left(x_{i}^{k}, x_{i}^{k-1}\right) \text {. }
$$

como $x_{i}^{k-1} \rightarrow \bar{x}_{i}>0, d$ es continuamente diferenciable en $\mathbb{R}_{++}^{n} \mathrm{y} \nabla_{1} d(x, x)=0$ obtenemos

$$
\lim _{k \rightarrow+\infty} \frac{\partial d_{i}}{\partial x_{i}}\left(x_{i}^{k}, x_{i}^{k-1}\right)=\frac{\partial d_{i}}{\partial x_{i}}\left(\bar{x}_{i}, \bar{x}_{i}\right)=0 .
$$

Ahora, usando in (3.9) la acotación de $\left\{\lambda_{k}\right\}$ y del anterior resultado obtenemos $\lim _{k \rightarrow+\infty} g_{i}^{k}=0$, para $i \in J(\bar{x})$.

Finalmente, probaremos iii). Sea $i \in J(\bar{x})$, entonces de la prueba de ii) tenemos que $\lim _{k \rightarrow+\infty} g_{i}^{k}=0$, así concluimos que $\lim _{k \rightarrow+\infty} g_{i}^{k} x_{i}^{k}=0$.

\section{Modelos Económicos}

Los modelos donde podemos aplicar el método de punto proximal aparecen en diversas líneas de investigación como por ejemplo, teoría de localización, optimización fraccionaria, teoría de decisiones y especialmente economía. En este artículo damos dos ejemplos, uno de ellos es un modelo de microeconomía ( que viene de la teoría del consumidor) y el otro es de la teoría de decisión proximal.

Ejemplo 3.1(Teoría económica de la decisión). Los modelos matemáticos en la teoría económica de la decisión consisten en la elección óptima de un agente económico (una empresa, un gobierno, un estado, etc.) dentro de todas las posibles alternativas a escoger. Hay tres elementos que caracterizan el problema de decisión del agente:

- El conjunto de elección, que nos dice cual es el universo de alternativas.

- El criterio de valorización, que estudia la manera en la que el agente evalúa las diferentes alternativas que se le ofrecen. Este criterio se define mediante una relación binaria reflexiva y transitiva , interpretada económicamente como "es al menos tan preferible" que refleja sus preferencias sobre el conjunto de elección. En economía esta relación es llamada preferencia.

- Las restricciones, que delimitan el conjunto de oportunidades sobre el cual el agente puede efectivamente elegir. 
Dado el conjunto de elección $X$, decimos que $\mu: X \rightarrow \mathbb{R}$ es una función de utilidad para $\precsim$ si para todo $x, y \in X$

$$
y \precsim x \text {, si y sólo si } \mu(y) \leq \mu(x) .
$$

Las preferencias $\precsim$ bajo ciertas condiciones sobre $X$ pueden representarse por una función de utilidad $\mu$. Así, elegir la mejor alternativa se convierte en encontrar una alternativa que maximice la función $\mu$. Por lo tanto concluimos que resolver el problema de decisión del agente es equivalente a resolver el problema de maximización:

$$
\operatorname{máx}\{\mu(x): x \in X\} .
$$

Un tipo particular de función de utilidad es la función cuasi-cóncava, esto es, $\mu$ satisface:

$$
\mu(\lambda x+(1-\lambda) y) \geq \min \{\mu(x), \mu(y)\}, \text { para todo } x, y \in X ; \text { y todo } \lambda \in[0,1] .
$$

Se puede probar que esta función está íntimamente relacionada a la hipótesis de convexidad de la preferencia, esto es, $\precsim$ es convexa si dados $x, y \in X$ con $x \precsim y$ y $x \precsim z$ en $X$ y $0 \leq \alpha \leq 1$ entonces

$$
x \precsim \alpha y+(1-\alpha) z .
$$

En términos económicos esta definición se interpreta como la tendencia natural del agente a diversificar su elección entre todas las mejores posibles, esto es, si $z$ e $y$ son dos alternativas al menos tan preferibles que $x$, entonces es natural pensar que la combinación $\alpha y+(1-\alpha) z$, para todo $\alpha \in[0,1]$, continua siendo al menos tan preferible que $x$.

La condición de convexidad de la preferencia $\precsim$ y algunas otras condiciones apropiaclas sobre $X$ y $\precsim$ permiten garantizar la existencia de una función de utilidad $\mu$ cuasi-cóncava y por lo tanto tener un problema de optimización cuasi-cóncavo (problemas donde la función objetivo es cuasi-cóncava y las funciones que definen las restricciones son cuasi-cóncavas).

Un problema particular es dado en el siguiente ejemplo del problema de la producción.

Considere que una empresa puede producir $n$ productos en cantidades $x_{1}, x_{2}, \ldots, x_{n}$, siendo sus costos de producción (por unidad) respectivamente igual a $p_{1}, p_{2}, \ldots, p_{n}$, donde $p_{i}>0$, para todo $i=1,2, \ldots, n$. Si se tiene un capital de $b$ soles para realizar la producción y una función continua $f$ que representa la utilidad de producción de los $n$ productos, el probleinci de conocer una utilidad mínima o máxima es expresado por

$$
\operatorname{opt}\left\{f(x): p^{T} x \leq b, x_{i} \geq 0\right\} .
$$

Ejemplo 3.2 (Decisión proximal, ver [5]). Sea el estado $X=\mathbb{R}_{+}^{n}$, interpretado económicamente como el conjunto de alternativas, ejecuciones, acciones, decisiones, etc. Un agente tiene una función objetivo $g: X \rightarrow \mathbb{R}$, que representa la ganancia por unidad de tiempo (utilidad, pago, ingreso, beneficio).

Supongamos que el supremo de este objetivo es finito, esto es, $\bar{g}=\sup \{g(x), x \in X\}<+\infty y$ asumamos que el agente comienza de algún estado inicial $x_{0}=x \in X$, conoce el supremo de $g$ y el valor inicial $g(x)=g\left(x_{0}\right)$. Así, él conoce cuan lejos está del supremo. Esta diferencia o llamada también salto es definida por

$$
n(x)=\bar{g}-g(x)
$$

La manera más natural de que el agente alcance su meta es encontrar el objetivo de manera progresiva dividiendolo por periodos que detallamos a continuación.

i. Fase exploratoria: El agente explora en un entorno de $x$, denotado por:

$$
E(x, r(x)) \subset X
$$

durante algún tiempo $\tau>0$, para descubrir $y \in E(x, r(x)) \subset X$. 
ii. Fase desplazamiento: El agente debe beneficiarse al obtener un nuevo estado mejorado. Él escoge explotar su beneficio en un tiempo $\delta>0$.

iii. Fase explotación: El agente debe beneficiarse al obtener un nuevo estado mejorado. Él escoge explotar su beneficio en un tiempo $\mu>0$.

Es así que del estado $x \in X$ descubrimos un nuevo estado $y \in X$ mejorado, tal que $g(y)>g(x)$, de modo que $n(y)<n(x)$.

En cada periodo $k \in \mathbb{N}$, se considera la función ganancia proximal

$$
P_{k}\left(x^{k}, y\right)=g(y)-\lambda_{k} C\left(x^{k}, y, \delta_{k}\right)
$$

donde $x^{k}, y \in E\left(x^{k}, r\left(x^{k}\right)\right), \lambda_{k}=\frac{1}{\mu_{k}}, C\left(x^{k}, y, \delta_{k}\right)$ es el costo de desplazamiento satisfaciendo $C\left(x^{k}, x^{k}, 0\right)=0$.

Así, el agente tratará de maximizar su ganancia proximal resolviendo para cada $k$

$$
x^{k+1} \in \operatorname{argmax}\left\{P_{k}\left(x_{k}, y\right): y \in E\left(x^{k}, r\left(x^{k}\right)\right)\right\},
$$

el cual es equivalente a:

$$
x^{k+1} \in \operatorname{argmin}\left\{n(y)+\lambda_{k} C\left(x^{k}, y, \delta\right): y \in E\left(x^{k}, r\left(x^{k}\right)\right)\right\} .
$$

El objetivo de este modelo es generar una sucesión $\left\{x^{k}\right\}$ tal que en un número finito de pasos o en el límite se obtenga la meta propuesta.

\section{Discusiones}

Se puede considerar este artículo como un avance importante en la extensión del método del punto proximal para resolver problemas de optimización no convexa. Estudios recientes encuentran modelos no convexos que son más aproximados de la realidad es por eso que algoritmos para tales problemas son de gran importancia para la optimización.

\section{Conclusiones}

- Se presenta algunos resultados de convergencia de una extensión del método del punto proximal para problemas de optimización no convexas con restriciones no negativas.

- Los modelos económicos que se modelan por funciones cuasi-convexas son dificiles de resolver usando técnicas de optimización conocidas es por eso que el aporte clado en este artículo puede ser considerado como una contribución para obtener algoritmos eficientes.

- Se espera en futuros trabajos dar condiciones de convergencia más fuertes del método presentado así como su implementación computacional.

\section{Agradecimientos}

Los autores agradecemos al Instituto de Investigación de la Facultad de Ciencias Matemáticas (UNMSM) por la oportunidad brindada para someter el presente artículo. 


\section{REFERENCIAS BIBLIOGRÁFICAS}

[1] Auslender A., Teboulle M. (2006) Interior gradient and proximal methods for convex and conic optimization. SIAM J. Optim., Vol. 16, 3: 697-725, 2006.

[2] Izmailov A. and Solodov M., Otimização Volume 1, IMPA, Rio de Janeiro, Brazil, 2005.

[3] Makela, M.M. Nonsmooth Optimization. Universitat Jyvaskyla: Mathematisches Institut, Bericht 47- April 1990.

[4] Rockafellar, R.T., Wets, R., Variational Analysis. Grundlehren der Mathematischen. Wissenschaften, 317, Springer, 1990.

[5] Souza S., Oliveira P.R., Cruz Neto J.X. and Soubeyran A., A Proximal Method with Separable Bregman Distance for Quasiconvex Minimization on the Nonnegative. European Journal of Operational Research, 201, 365-376, 201(). 